\title{
VIPoma: an unsual cause of electrolyte disturbance
}

Diana Boj Carceller, Diego Álvarez Ballano, María Eugenia López Valverde, Beatriz Lardiés Sánchez, Leticia Pérez Fernández, Isabel Azcona, Alejandro Sanz Paris, Javier Acha Pérez

Department of Endocrinology \& Nutrition. Hospital Universitario Miguel Servet. Zaragoza. Spain

\section{Introduction}

Vasoactive intestinal peptide-producing tumours (VIPomas) represent a rare type of neuroendocrine tumour whose incidence is 1 in 10 million per year. Most are located in the pancreas. They cause diffuse watery (secretory) diarrhoea, hypokalaemia and achlorhydria and also appear as an uncommon cause of hypercalcemia.

\section{Case Report}

A 69-year-old female with previous diagnosis of a "non-functioning" neuroendocrine pancreatic tumor (2005) with liver metastasis (2009) was admitted for weakness. She complained of chronic watery diarrhoea that persisted with fasting (6-10 stools/day) in spite of multiple therapies. She had been admitted for hypercalcemia a few months before. Her family history was negative for MEN-1 affected.

At admission: glucose $159 \mathrm{mg} / \mathrm{dL}$, creatinine $0.82 \mathrm{mg} / \mathrm{dL}, \mathrm{K}+2.6 \mathrm{mEq} / \mathrm{L}, \mathrm{Na}+128$ $\mathrm{mEq} / \mathrm{L}, \mathrm{Cl}-102 \mathrm{mEq} / \mathrm{L}, \mathrm{pH} 7.34$, serum bicarbonate $15.1 \mathrm{mmol} / \mathrm{l}, \mathrm{P} 1$ $\mathrm{mg} / \mathrm{dL}$, Mg $1.6 \mathrm{mg} / \mathrm{dL}$, Ca $15.3 \mathrm{mg} / \mathrm{dl}, \mathrm{Hb} 12.8 \mathrm{~g} / \mathrm{dL}, \mathrm{GGT} 131 \mathrm{U} / \mathrm{L}, \mathrm{PA} 93$ $\mathrm{U} / \mathrm{L}$, AST $44 \mathrm{U} / \mathrm{L}$, GPT $28 \mathrm{U} / \mathrm{L}$. Albumin $4.1 \mathrm{~g} / \mathrm{dL}$.

Serum TSH, calcitonin, cortisol, PRL, glucagon, PTH-rp and 5HIAA were normal. PTH $23.8 \mathrm{pg} / \mathrm{mL}$. 25-OH-vitamin D $35.6 \mathrm{nmol} / \mathrm{L}$. CgA 115 $\mathrm{ng} / \mathrm{mL} . \mathrm{VIP}>116 \mathrm{pmol} / \mathrm{L}(<30)$. Gastrin $149 \mathrm{pg} / \mathrm{ml}$ (13-115).
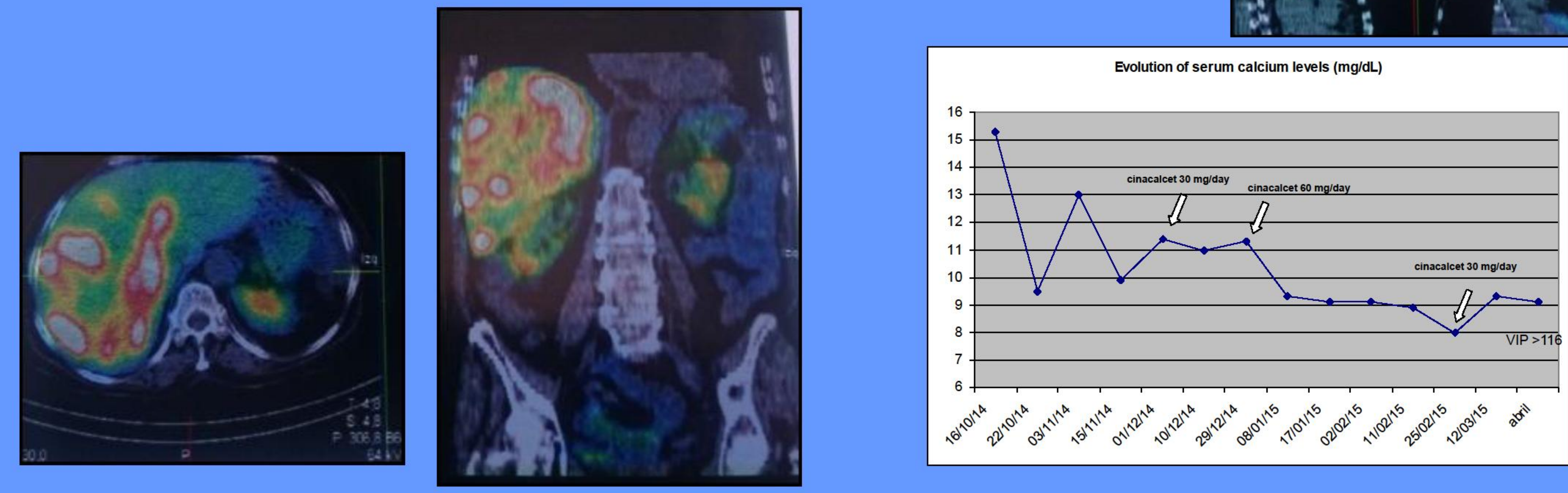

Endocrinological treatment: potassium 125-200 mEq/d, phosphorus $32-48 \mathrm{mmol} / \mathrm{d}$, magnesium 4-8 mEq/d, high intake of salt and water, insulin 20-40 u/d, periodic iv biphosponates, PPI $20 \mathrm{mg} / \mathrm{d}$, octreotide LAR $30 \mathrm{mg} / 28 \mathrm{~d}$, LT4 $25 \mathrm{mcg} / \mathrm{d}$, cinacalcet $30-60 \mathrm{mg} / \mathrm{d}$

\section{Discussion}

- VIPomas may initially present as non-funtioning NETs.

- These are a rare cause of hypercalcemia. This might be partially explained by the stimulatory effect of VIP on bone resorption. The other electrolyte disturbances may be attributed to the severe diarrhoea.

- As a new fact, we present cinacalcet as a drug to be considered in hypercalcemia related to VIPoma refractory to conventional therapy

References:

1. Wägner AM, Trías M, Campo R, López J, Rodríguez M, Webb SM. Pancreatic vasoactive intestinal peptide-producing tumor and hypercalcemia. Eur J Intern Med. 2004;15:328

2. Ahmad S, Kuraganti G, Steenkamp D. Hypercalcemic crisis: a clinical review. Am J Med. 2015;128:239-45.

3. El-Khouly F, Khan MS, Toumpanakis C, Meyer T, Caplin M. Challenges in the Management of Vipoma Patients. Gastroenterology. 2011;140:S-876 